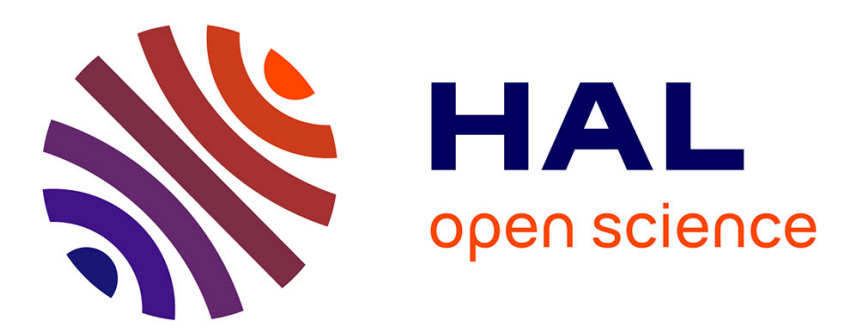

\title{
Contribution à l'étude des capteurs magnétiques à induction pour la prospection magnéto-tellurique étude de la sensibilité et améliorations
}

\author{
Y. Benderitter, L. Le Donche
}

\section{- To cite this version:}

Y. Benderitter, L. Le Donche. Contribution à l'étude des capteurs magnétiques à induction pour la prospection magnéto-tellurique étude de la sensibilité et améliorations. Revue de Physique Appliquée, 1970, 5 (1), pp.183-185. 10.1051/rphysap:0197000501018300 . jpa-00243357

HAL Id: jpa-00243357

https://hal.science/jpa-00243357

Submitted on 1 Jan 1970

HAL is a multi-disciplinary open access archive for the deposit and dissemination of scientific research documents, whether they are published or not. The documents may come from teaching and research institutions in France or abroad, or from public or private research centers.
L'archive ouverte pluridisciplinaire HAL, est destinée au dépôt et à la diffusion de documents scientifiques de niveau recherche, publiés ou non, émanant des établissements d'enseignement et de recherche français ou étrangers, des laboratoires publics ou privés. 


\title{
GONTRIBUTION A L'ÉTUDE DES GAPTEURS MAGNÉTIQUES A INDUGTION POUR LA PROSPEGTION MAGNÉTO-TELLURIQUE
}

\section{ÉTUDE DE LA SENSIBILITÉ ET AMÉLIORATIONS}

\author{
Par Y. BENDERITTER et L. LE DONGHE, \\ Centre de Recherches Géophysiques, 58-Garchy (France).
}

\begin{abstract}
Résumé. - L'enregistrement des variations du champ magnétique terrestre demande des capteurs aux performances de plus en plus élevées. Le but de la première partie de cet exposé consiste à définir à partir du phénomène la sensibilité nécessaire à un bon enregistrement et à voir comment on peut améliorer cette sensibilité d'une façon générale. Dans la seconde partie, on développe un des moyens d'augmenter cette sensibilité ainsi qu'une modification permettant d'alléger le noyau.
\end{abstract}

Abstract. - The recording of variations in the earth's magnetic field calls for receivers with higher and higher standards of performance. The first part of this article is intended to define, for a given phenomenon, the sensitivity required for a good recording and to see how this sensitivity can be improved generally. In the second part, one way of increasing this sensitivity is discussed in detail, together with a modification making it possible to lighten the core.

Cet exposé comprend deux parties : la première concerne l'étude de la sensibilité de capteurs magnétiques utilisés en prospection géophysique (Benderitter) et la seconde est relative à des modifications et à des améliorations de ces capteurs (Le Donche).

1. Sensibilité. - La prospection magnéto-tellurique demande des capteurs très sensibles car l'amplitude des variations du champ magnétique à mesurer est parfois très petite. Les valeurs moyennes relevées lors d'un sondage sur le terrain sont données en fonction de la période $T$ sur la figure 1 (courbe « Champ

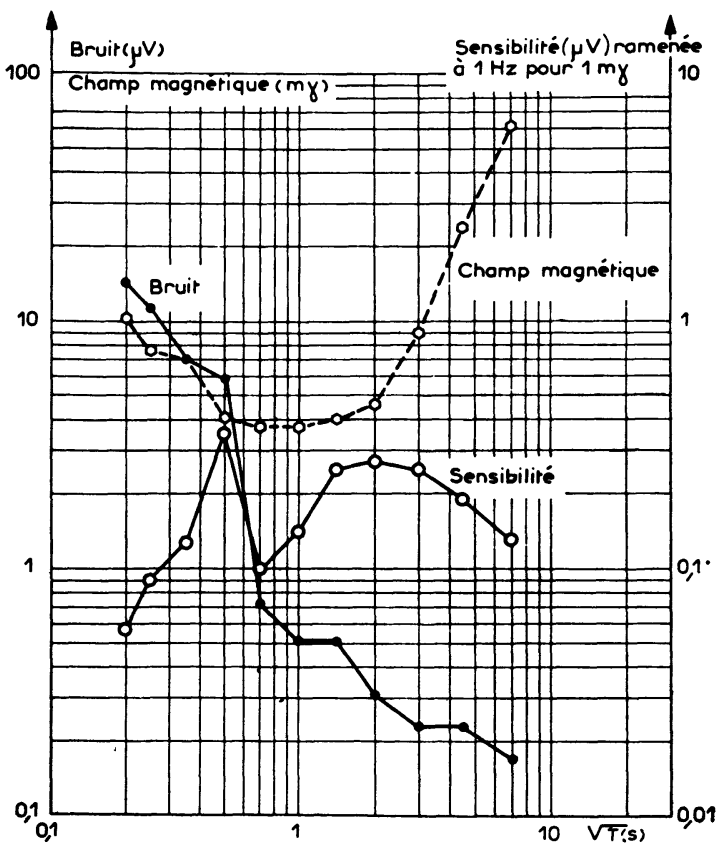

FIG. 1. magnétique» avec valeurs exprimées en milligammas). Les phénomènes étant imposés, le problème revient donc à convertir ces milligammas en une grandeur convenablement mesurable. Les capteurs à induction semblaient indiqués surtout pour les faibles périodes (jusqu'à $0,01 \mathrm{~s}$ ). Ils se sont révélés encore valables pour les périodes les plus élevées du spectre à étudier (jusqu'à $900 \mathrm{~s}$ ).

a) Sensibilité Utile Requise. - Nous définirons ici la sensibilité utile comme étant la sensibilité pour laquelle le rapport signal/bruit est égal à l'unité. Dans le cas présent, le bruit provient surtout des premiers circuits électroniques. Son amplitude, ramenée à la sortie de l'enroulement, est représentée aussi sur la figure 1 (courbe « Bruit » avec valeurs exprimées en microvolts). Amplitudes du phénomène et du bruit permettent alors de calculer la sensibilité utile pour les différentes périodes. Les valeurs sont données par la troisième courbe de la figure 1 (courbe «Sensibilité» avec valeurs exprimées en microvolts par milligamma et par hertz). Retenons la valeur la plus difficile à atteindre :

$$
0,35 \mu \mathrm{V} / \mathrm{m} \gamma / \mathrm{Hz} \text {. }
$$

Les capteurs devront être construits en fonction de cette sensibilité, mais on verra que la fréquence de résonance intervient aussi. Étant destinés à la prospection, les capteurs doivent être légers et d'encombrement réduit. L'emploi d'un noyau devient appréciable car il multiplie le flux d'induction par :

$$
\frac{s}{S} \frac{\mu}{1+\frac{\mu}{8(l / d)^{2}}}
$$

$s, l$ et $d$ étant la section, la longueur et le diamètre du noyau, $S$ la section moyenne de l'enroulement et $\mu$ la perméabilité du noyau. Les fortes valeurs de $l / d$ sem- 
blent intéressantes car la relation tend vers $\mu s / S$. Malheureusement, ce coefficient n'est pas constant puisqu'il dépend de la perméabilité qui est un paramètre variable (on peut d'ailleurs exploiter cette propriété mais dans un montage tout à fait différent, en incluant par exemple le bobinage du capteur dans un circuit oscillant de façon à obtenir un signal modulé par les variations du champ magnétique). Il est donc préférable de prendre de faibles valeurs pour $l / d$. La relation précédente tend alors vers $2 \pi l^{2} / S$ et le signal recueilli devient proportionnel à $n l^{2}, n$ étant le nombre de spires du bobinage. Une confirmation en est donnée par les résultats expérimentaux de la figure 2

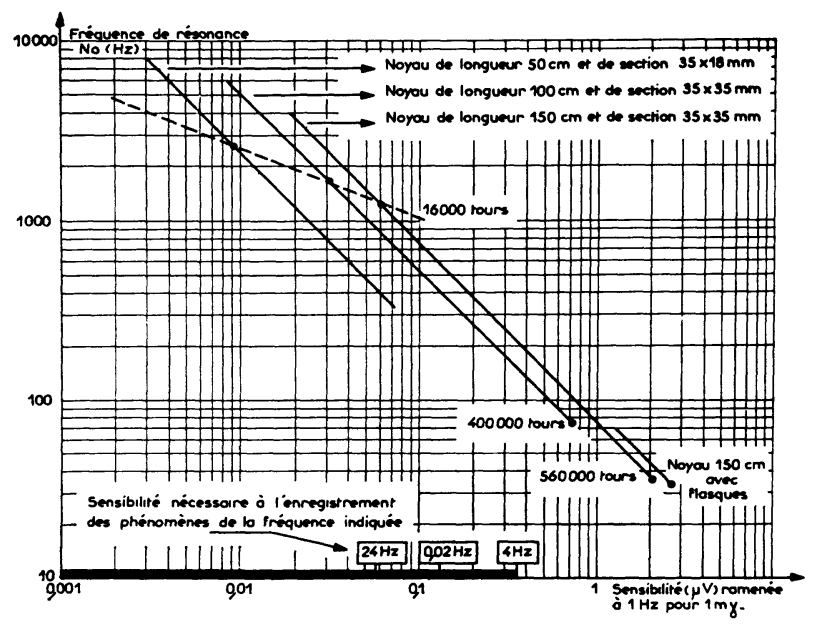

FIG. 2.

sur laquelle chaque droite donne la sensibilité, repors tée sur l'axe des abscisses, suivant le nombre de spireet pour un noyau de longueur donnée.

Sur cette figure, on peut voir aussi que la sensibilité utile $\sigma$ n'est obtenue que pour un nombre élevé de spires, c'est-à-dire pour une impédance importante et par conséquent avec une fréquence de résonance $N_{0}$ relativement faible, fréquence qui est donnée suivant l'axe des ordonnées. Or il est préférable de rejeter cette fréquence en dehors du spectre à étudier. Ce qui paraît impossible puisque, comme le montrent les résultats expérimentaux, la sensibilité est inversement proportionnelle à $N_{0}$ pour un noyau et un bobinage de type donné. Heureusement la sensibilité utile diminue pour les fréquences supérieures à $4 \mathrm{~Hz}$, ce qui permet de réduire alors le nombre de spires quand on étudie les fréquences élevées et d'augmenter d'autant $N_{0}$.

b) Capteurs réalisés pour la prospegtion. - Les capteurs de prospection que nous employons le plus couramment (voir fig. 3) sont composés d'un noyau en mu-métal de longueur $150 \mathrm{~cm}$ et de section carrée de $12 \mathrm{~cm}^{2}$ sur lequel sont disposées 560000 spires réparties en 70 bobines. Leur sensibilité est de $2,6 \mu \mathrm{V} / \mathrm{m} \gamma / \mathrm{Hz}$ et leur fréquence de résonance de $34 \mathrm{~Hz}$. Pour l'étude des fréquences élevées, le nombre de spires est divisé par 5 .

L'étude des variations du champ magnétique suivant la verticale et les mesures en mer demandent des capteurs plus courts. Nous en avons réalisé (voir fig. 4). Leurs caractéristiques sont : $l=100 \mathrm{~cm}$,

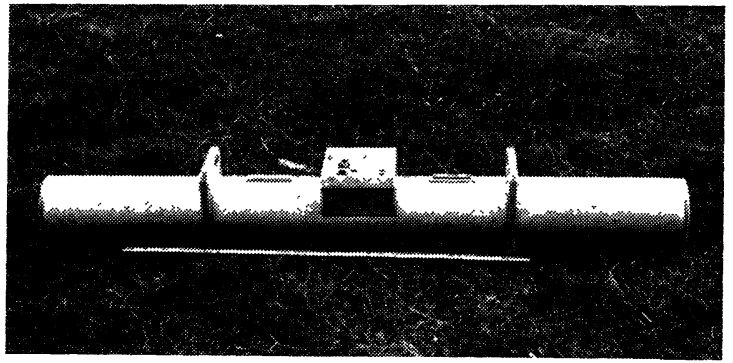

FIG. 3.

$n=400000$ spires, $\sigma=1 \mu \mathrm{V} / \mathrm{m} \gamma / \mathrm{Hz}$ et $N_{0}=80 \mathrm{~Hz}$.

Pour l'étude des fréquences supérieures à $100 \mathrm{~Hz}$, Dupis a réalisé un capteur dont les caractéristiques ( $l=40 \mathrm{~cm}, s=64 \mathrm{~mm}^{2}$ et $n=160000$ ) assurent une sensibilité de $0,065 \mu \mathrm{V} / \mathrm{m} \gamma / \mathrm{Hz}$.

Tous ces capteurs bénéficient des deux améliorations qui seront développées plus loin. Ils comportent aussi un circuit électronique de faible consommation. Ce circuit que l'on peut voir sur la figure 4 délivre à sa sortie un signal amplifié sous une faible impédance.

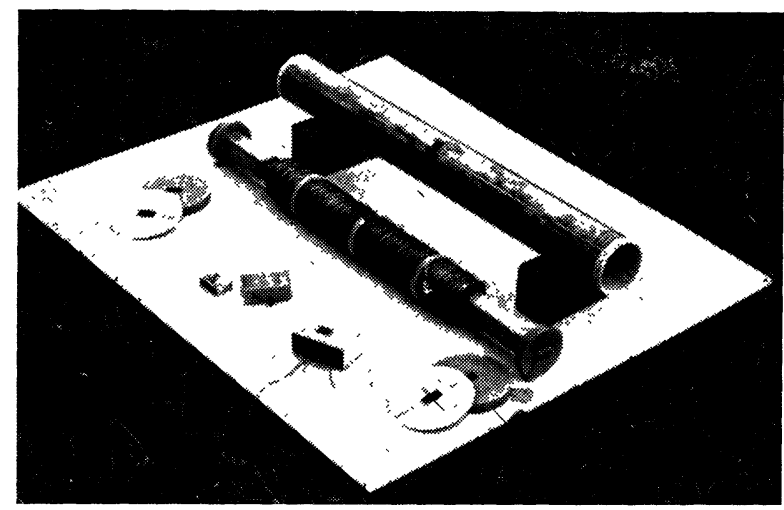

FIG. 4.

Pour avoir un meilleur rapport signal/bruit, on peut soit augmenter le signal en accroissant la sensibilité, soit diminuer le bruit. Dans le second cas, l'évolution de l'électronique permettra de diminuer encore le bruit du circuit incorporé au capteur. Cependant, les progrès dans cette voie seront limités par l'apparition d'autres sources de bruit. Dans le premier cas, augmenter la longueur du noyau ou le nombre de spires est peu recommandé et parfois impossible. On peut essayer aussi de modifier l'enroulement pour obtenir un produit $N_{0} \sigma$ plus grand. Un autre moyen va être développé maintenant.

2. Modifications et améliorations. - Il est donc souhaitable pour la prospection d'améliorer la sensibilité du capteur sans en augmenter les dimensions et le poids. Le problème consiste à trouver un moyen de concentrer les lignes de force du champ magnétique sans modifier surtout la longueur du noyau. Pour cela, il paraissait intéressant d'augmenter le volume de mu-métal aux extrémités du noyau. Cela a été réalisé de façon très pratique en ajoutant un ou plusieurs disques de mu-métal (voir fig. 4), disques dont les axes sont confondus avec celui du noyau. La figure 5 donne le gain obtenu pour des noyaux de 


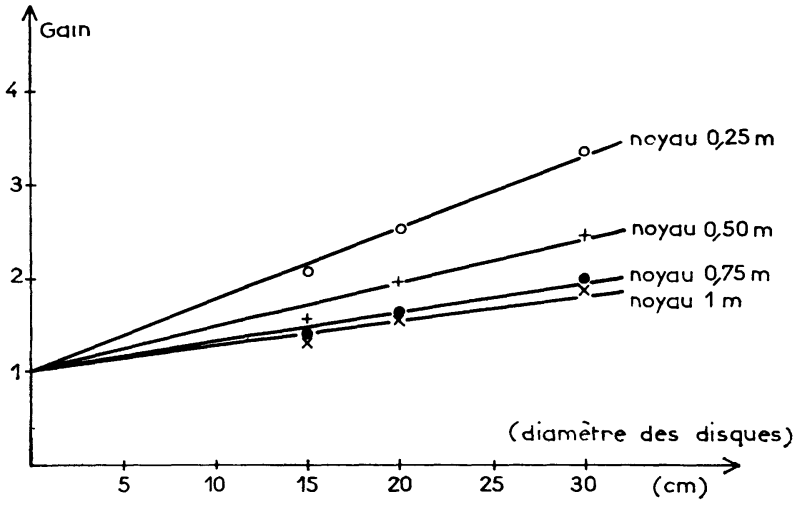

FIG. 5.

diverses longueurs, munis de disques de diamètres différents et de $1 \mathrm{~mm}$ d'épaisseur. On peut remarquer qu'il est plus facile d'augmenter le gain, par ce moyen, quand le noyau est court. Pour des raisons pratiques, les capteurs courants de prospection ont été munis de disques de $15 \mathrm{~cm}$ de diamètre seulement. Par contre, il n'y a que des avantages à avoir de plus grands disques pour les capteurs verticaux, leur stabilité en position n'en étant que meilleure.

L'adjonction de disques augmentant légèrement le poids du capteur, il convenait de chercher un moyen de le diminuer d'un autre côté. L'auteur a étudié la variation de sensibilité quand on supprime du mumétal au centre du noyau, ce qui est facilement réalisable puisque les noyaux sont constitués d'un empilement de lamelles. La figure 6 illustre les résultats obtenus avec un noyau de $40 \mathrm{~cm}$ de longueur dans la gamme de $20 \mathrm{~Hz}$ à $1500 \mathrm{~Hz}$. La courbe (a) correspond à un noyau plein, de section $5,5 \mathrm{~cm}^{2}$, et la courbe (b) correspond à un noyau creux de section intérieure $6,6 \mathrm{~cm}^{2}$ et de section extérieure $7,3 \mathrm{~cm}^{2}$. Les courbes $\left(a^{\prime}\right)$ et $\left(b^{\prime}\right)$ correspondent aux résultats obtenus avec les noyaux précédents après adjonction

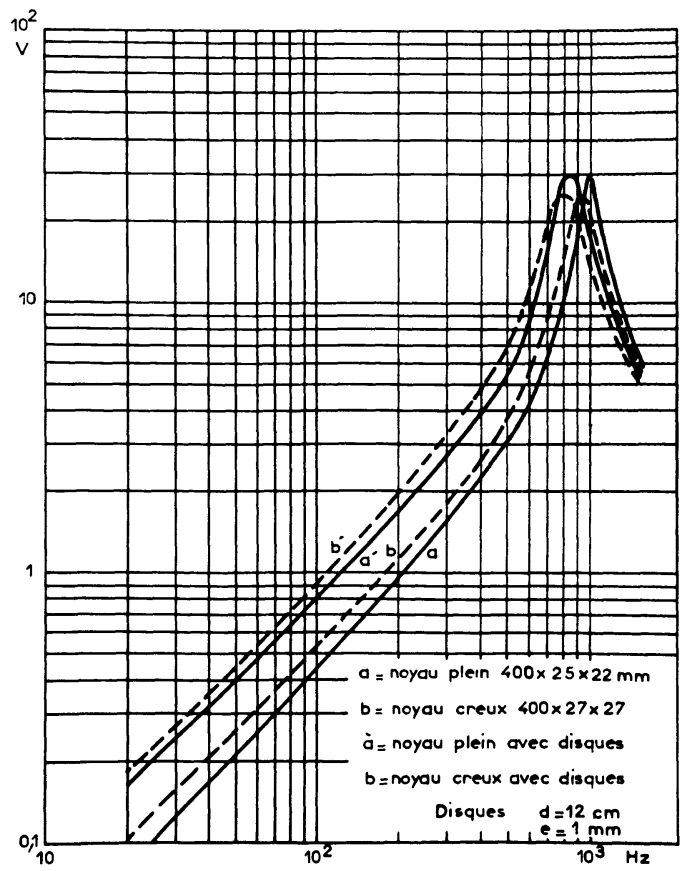

FIG. 6.

de disques de $12 \mathrm{~cm}$ de diamètre et de $1 \mathrm{~mm}$ d'épaisseur. L'amplitude du signal est multipliée par 1,7 et la fréquence de résonance diminue seulement de $15 \%$.

Les capteurs à induction ont permis de résoudre notre problème. Les deux modifications précédentes, très facilement réalisables du point de vue mécanique, ont entraîné une amélioration appréciable en augmentant leur sensibilité et en diminuant leur poids. Cependant, les progrès restent limités et si l'on veut des performances beaucoup plus élevées, il faudra sans doute abandonner cette solution classique et attendre son remplacement par un autre type de capteurs. 